\title{
Weighted correlation network analysis of triple-negative breast cancer progression: Identifying specific modules and hub genes based on the GEO and TCGA database
}

\author{
LEI LAN ${ }^{1 *}$, BIN XU ${ }^{2,3^{*}}$, QU CHEN ${ }^{1}$, JINGTING JIANG $^{2,3}$ and YUEPING SHEN ${ }^{1}$ \\ ${ }^{1}$ Jiangsu Key Laboratory of Preventive and Translational Medicine for Geriatric Diseases, \\ Department of Epidemiology and Biostatistics, School of Public Health, Soochow University, Suzhou, Jiangsu 215123; \\ ${ }^{2}$ Department of Tumor Biological Treatment; ${ }^{3}$ Jiangsu Engineering Research Center for Tumor Immunotherapy, \\ The Third Affiliated Hospital of Soochow University, Changzhou, Jiangsu 213000, P.R. China
}

Received May 16, 2018; Accepted January 16, 2019

DOI: $10.3892 / \mathrm{ol} .2019 .10407$

\begin{abstract}
Triple-negative breast cancer (TNBC) represents an aggressive malignancy of frequent high histologic grade with no effective specific targeted therapies. The present study aimed to identify specific modules and hub genes that may influence the progression of TNBC. The key words 'breast cancer' were used to search microarray datasets in the Gene Expression Omnibus and The Cancer Genome Atlas databases that included 5 datasets. A total of 11 co-expression modules were constructed based on the expression levels of 5,782 genes obtained from 456 patients with TNBC using the weighted correlation network analysis (WGCNA). The results demonstrated that the red module was significantly associated with relapse-free survival (RFS) in patients with TNBC [hazard ratio $(\mathrm{HR})=0.381,95 \%$ confidence interval $(\mathrm{CI}), 0.183-0.793$; $\mathrm{P}=0.010]$. The functional enrichment analysis revealed that the biological processes corresponding to the red module were 'mRNA processing', 'histone lysine methylation' and 'regulation of TOR signaling'. In addition, Hedgehog signaling pathways were considered to serve a critical role in the development of this disease $(\mathrm{P}<0.001)$. A total of 12 hub genes were identified, of which $\alpha$-thalassemia/mental retardation syndrome X-linked (ATRX) was significantly associated with RFS in patients with TNBC $(\mathrm{HR}=0.601 ; 95 \% \mathrm{CI}, 0.376-0.960$;
\end{abstract}

Correspondence to: Professor Yueping Shen, Jiangsu Key Laboratory of Preventive and Translational Medicine for Geriatric Diseases, Department of Epidemiology and Biostatistics, School of Public Health, Soochow University, 199 Ren-Ai Road, Suzhou, Jiangsu 215123, P.R. China

E-mail: shenyueping@suda.edu.cn

*Contributed equally

Key words: triple-negative breast cancer, weighted gene co-expression network analysis, progression, hub genes, $\alpha$-thalassemia/mental retardation syndrome $\mathrm{X}$-linked
$\mathrm{P}=0.033$ ). The receiver operating characteristic curve indicated that ATRX could distinguish relapse from non-relapse in patients with TNBC (area under the curve $=0.570 ; \mathrm{P}=0.023$ ). In conclusion, the present study demonstrated that ATRX was associated with TNBC progression, which suggested that ATRX may be involved in a recombination-mediated telomere maintenance mechanism.

\section{Introduction}

Breast cancer is the most commonly diagnosed cancer in women and the leading cause of cancer-associated mortality worldwide. In 2018, 41,400 patients succumbed to this malignancy in the United States. Estimations highlight that 268,670 new cases may be diagnosed every year (1). Triple-negative breast cancer (TNBC) represents 10-20\% of all breast cancer cases and is defined by a lack of estrogen receptor (ER) and progesterone receptors (PR) expression and the absence of amplification or overexpression of human epidermal growth factor receptor 2 (HER2) (2). In addition, TNBC is associated with a higher risk of distant and early recurrence and more aggressive metastases in the viscera and central nervous system, particularly in the lungs and brain (3). Due to the lack of specific targeted therapies in TNBC, endocrine or anti-HER2 therapies display no benefits, and chemotherapy is the only established therapeutic option available in clinical practice (3-6). It is therefore crucial to identify and develop specific molecular targets for the development of effective treatment of TNBC.

With the development of genomic technologies, a large volume of molecular information including Gene Expression Omnibus (GEO) and The Cancer Genome Atlas (TCGA), represent a remarkable opportunity to analyze the gene expression data for the discovery of novel targets (7). Furthermore, co-expression analysis has emerged as a powerful technique for multigene analysis in large-scale data. Gene co-expression analysis is used to associate genes of unknown function with biological processes, in order to prioritize candidate disease genes or to differentiate transcriptional regulatory programs (8). In comparison with the traditional 
one-dimensional molecular biology methods, the weighted correlation network analysis (WGCNA) is a method that can highlight the modules of highly correlated genes and describe the characteristics of the biological system more accurately and effectively $(9,10)$. This method has been successfully used to identify targeted modules and hub genes in cancer research. For example, Chen et al (11) and Wan et al (12) identified novel biomarkers for human clear renal cell carcinoma and uveal melanoma, respectively, via WGCNA. In addition, Clarke et al (13) used WGCNA to analyze a breast cancer dataset and identified certain modules associated with different molecular subtypes. In the present study, WGCNA has highlighted numerous biologically significant results in cancer study, and to the best of our knowledge, it was applied for the first time to the study of TNBC. WGCNA represents an $\mathrm{R}$ package for weighted correlation network analysis, including network construction, module detection, gene selection, topological property calculation and visualization (14). In the present study, WGCNA was used to construct a TNBC gene co-expression network. Firstly, the initial phase of the WGCNA method allowed identification of co-expression modules. Secondly, a survival analysis for modules identified in the first step was performed. Thirdly, a functional enrichment analysis was achieved on the modules of interest. The identified hub genes may therefore be beneficial to assess the malignancy and prognosis of TNBC.

\section{Materials and methods}

Search strategy. In the present study, mRNA expression data and clinical trait information of patients with breast cancer were downloaded from the GEO database using the keywords 'breast cancer' in NCBI (http://www.ncbi.nlm.nih. gov/geo/) and TCGA database (https://cancergenome.nih. gov/, last updated July 2017). The search strategy of the study was designed as follows: The type of study was 'expression profiling by array', and the entry type was 'datasets'. The sample size of the selected datasets was $\geq 100$. The organism was homo sapiens. The database searching was independently carried out by two researchers.

Data preprocessing. Four microarray datasets (GSE16446, GSE25055, GSE25065 and GSE58812) that contained survival outcomes and clinical information of ER, PR and HER2 were selected from the GEO database. Data of samples from patients with TNBC were obtained from the TCGA database (Fig. 1). Table I presents the clinical characteristics of the patients with TNBC included in these five datasets. In addition, four endpoints were integrated and defined as relapse-free survival (RFS), which represented survival outcomes. The mRNA expression value of each gene considered in the present study represented the mean of the gene expression values extracted from the five datasets. The mRNA gene expression from the five datasets were normalized and merged by gene name; however, each gene that was not present in all datasets was excluded from the study. In order to ensure the quality of the expression data, genes were excluded if $\geq 10 \%$ of the samples were missing expression data. Subsequently, the variance of each mRNA gene expression value was calculated and the genes with variance ranked in the top $50 \%$ were selected. Eventually,
Table I. Clinical characteristics of patients with TNBC.

\begin{tabular}{lccc}
\hline Dataset & Patients, $n$ & Survival endpoints & Event $(0 / 1)$ \\
\hline TCGA & 83 & RFS & $69 / 14$ \\
GSE58812 & 107 & MFS & $76 / 31$ \\
GSE25065 & 64 & DRFS & $37 / 27$ \\
GSE25055 & 114 & DRFS & $77 / 37$ \\
GSE16446 & 45 & DMFS & $32 / 13$
\end{tabular}

Event 0, occurred; Event 1, did not occur. DMFS, distant meta-free survival; DRFS, distant relapse-free survival; MFS, meta-free survival; RFS, relapse-free survival; TCGA, The Cancer Genome Atlas.

Table II. Clinical characteristics of patients with TNBC.

\begin{tabular}{lc}
\hline Variables & Patients, $\mathrm{n}(\%)$ \\
\hline Age, years & \\
$\leq 50$ & $208(45.3)$ \\
$>50$ & $251(54.7)$ \\
Stage & \\
I & $23(7.8)$ \\
II & $162(55.1)$ \\
III & $108(36.7)$ \\
IV & $1(0.4)$ \\
Lymph Node & \\
N0 & $148(42.0)$ \\
N1 & $135(38.4)$ \\
N2 & $40(11.4)$ \\
N3 & $29(8.2)$ \\
T stage & \\
T1 & \\
T2 & $44(12.6)$ \\
T3 & $199(56.9)$ \\
T4 & $67(19.1)$ \\
Metastasis & $40(11.4)$ \\
Yes & \\
No & \\
OS ${ }^{a}$, months & $116(99.1)$ \\
RFS ${ }^{a}$, months & $1(0.9)$ \\
\hline
\end{tabular}

RFS represents all endpoints of survival information, including distant meta-free survival, distant relapse-free survival, meta-free survival and RFS. OS, overall survival; RFS, relapse-free survival; TNBC, triple-negative breast cancer. ${ }^{a} \mathrm{OS}$ and RFS describe the $\mathrm{P}_{50}$ $\left(\mathrm{P}_{25}, \mathrm{P}_{75}\right)$ of survival time.

a final dataset containing 459 patients with TNBC and mRNA expression of 5,782 genes was compiled. Table II presents the baseline clinical characteristics of the final dataset.

Construction of the WGCNA. The WGCNA was carried out by using the R WGCNA package (15). The goodSamplesGenes 


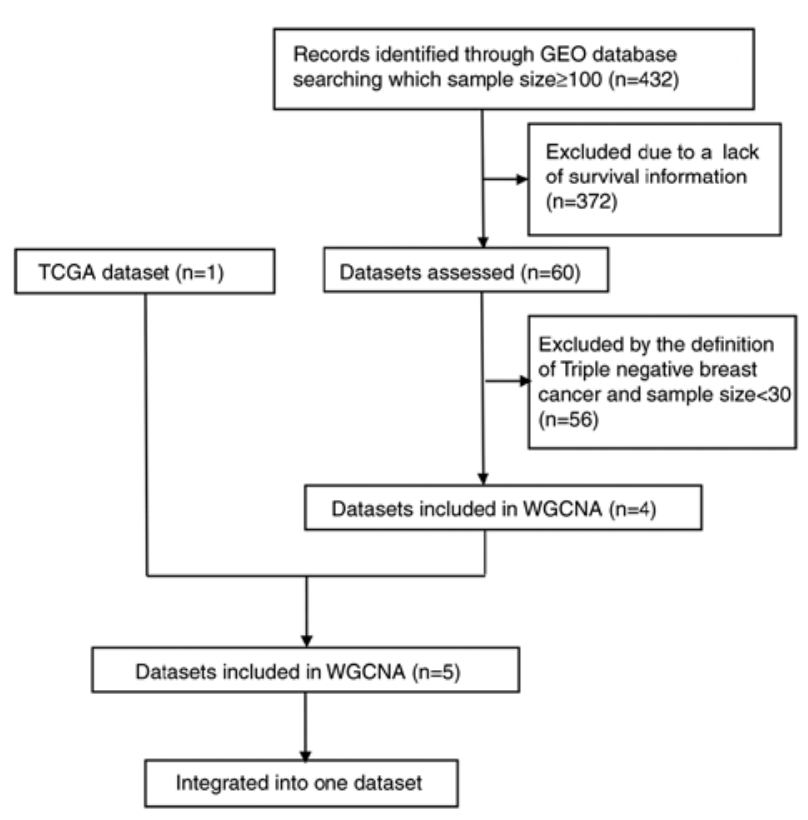

Figure 1. Flow diagram of dataset collection process. GEO, Gene Expression Omnibus; TCGA, The Cancer Genome Atlas; WGCNA, weighted correlation network analysis.

function in the R WGCNA package was used to check the gene expression data of all TNBC samples for excessive missing values and identification of outlier microarray samples. The samples were clustered with hierarchical clustering analysis by using the hclust function to check if there were any outliers. A correlation matrix was created by using a similarity measure to summarize the association between all genes. In addition, to identify specific modules, WGCNA uses a soft-thresholding procedure to avoid the selection of an arbitrary cut-off. The $\beta$ value represented a soft-thresholding parameter that could emphasize strong correlations between genes and penalize weak correlations to ensure a scale-free network (14). The cutreeDynamic function was used for adaptive branch pruning of hierarchical clustering dendrograms and the dynamicTreeCut package was adopted to generate co-expression modules. Subsequently, to further analyze the module, the dissimilarity of the module eigengenes (ME) was calculated using the moduleEigengenes function in the R WGCNA package, which was defined as the first principal component of a given module and considered to be representative of the gene expression profiles in a module. A cut-off line for the module dendrogram was selected and the module was merged. Eventually, the adjacency was converted into a topological overlap matrix (TOM), and modules were subjected to hierarchical cluster analysis according to the TOM-based dissimilarity measure.

To assess the potential associations between modules and clinical variables, approaches based on WGCNA to identify modules associated with to the progression of TNBC were used. Firstly, the gene significance (GS) was defined as the $\log 10$ transformation of the corresponding $\mathrm{P}$-value $(\mathrm{GS}=\lg \mathrm{P})$ of the correlation between gene expression and pathological stage. Secondly, each ME considered as the major component in the principal component analysis was chosen and represented the mean measure for the overall co-expression network. Ultimately, the correlation between MEs and the clinical characteristics was calculated to identify the relevant module (16-18).

Statistical analysis. For survival analysis, MEs and gene expression values were divided into low and high expression groups by using the Cutoff Finder (http://molpath.charite. de/cutoff/index.jsp) (19). The hazard ratio (HR) was determined via a Cox regression model, and survival curves were plotted from Kaplan-Meier estimations. $\mathrm{P}<0.05$ was considered to indicate a statistically significant difference.

Gene Ontology (GO) and Kyoto Encyclopedia of Genes and Genomes (KEGG) pathways enrichment analyses for the identified modules were performed using Cytoscape software (version 3.5.1; https://cytoscape.org/) with the ClueGO plug-in (version 2.5.0; http://apps.cytoscape.org/apps/cluego) (20). The ClueGO plug-in generates grouped GO annotation and KEGG pathways, and integrates the terms to create a functionally organized $\mathrm{GO} /$ pathway term network by using $\kappa$ statistics to determine the association strength between the terms (21). In the present study, the statistical test used by the ClueGO plug-in for the enrichment was based on two-sided hypergeometric tests with a Benjamini-Hochberg adjustment. GO terms and KEGG pathways with $\mathrm{P}<0.05$ were considered as significantly different, and a $\kappa$ score threshold $\geq 0.4$ was used to functionally divide these pathways into different groups.

Identification of hub genes in the co-expression module. WGCNA is used to find hub genes in the module of interest, which is highly interconnected with other genes that have higher biological relevance compared with the whole network. The absolute value of Pearson's correlation between gene expression and MEs was used to identify the importance of a gene in the module, which is known as the module membership (MM). In the present study, hub genes were selected for $\mathrm{MM}>0.55$ in the specific module. In addition, the modules of interest were constructed using Cytoscape and defined as hub genes for a connectivity degree $\geq 15$ in the co-expression network. The common hub genes with the higher MM and connectivity degree were considered as 'real' hub genes in the module of interest.

\section{Results}

Construction of the WGCNA. A total of 459 TNBC samples were used as input for the hierarchical clustering analysis that was performed with the function hclust to cluster the samples to see if there were any clear outliers. Three samples (GSM149983, GSM1419985 and GSM411317) were removed as outliers. The co-expression network was constructed from the expression values of 5,782 genes in 456 TNBC samples with the WGCNA package. Prior to further studying the TNBC samples, an analysis of network topology was performed for various soft-thresholding powers to obtain the relatively balanced scale independence and mean connectivity. As presented in Fig. 2A, power 3, which is the lowest power for which the scale-free topology fit index reached 0.90 , was selected to ensure a scale-free network and to produce a hierarchical clustering dendrogram. The dynamic tree cut function was used to prune the branches in hierarchical 

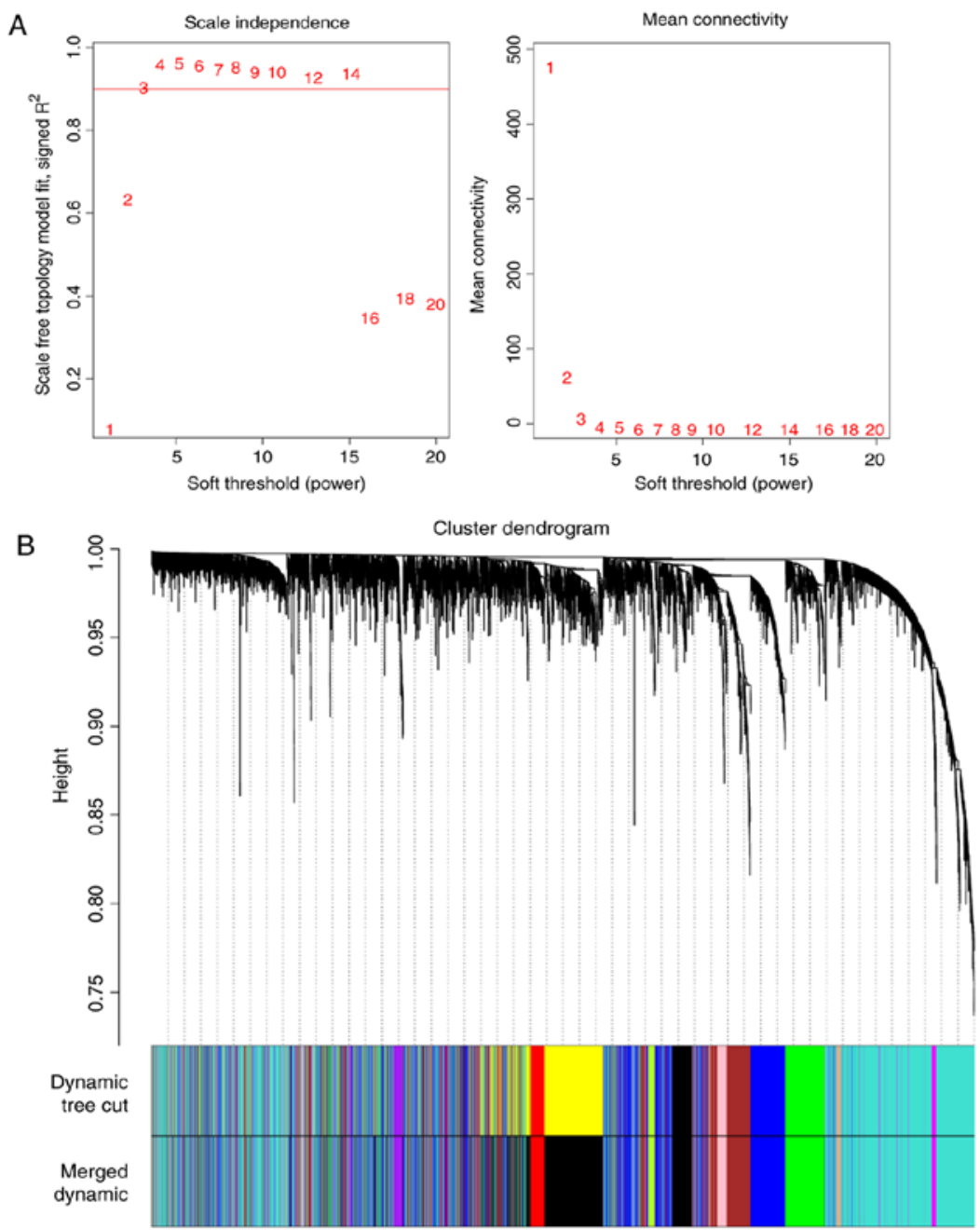

C

\begin{tabular}{|c|c|c|c|c|}
\hline Magenta & $-0.11(0.06)$ & $-0.04(0.50)$ & $-0.04(0.50)$ & $-0.05(0.40)$ \\
\hline arquoise & $0.00(0.90)$ & $-0.01(0.90)$ & $0.04(0.50)$ & $-0.09(0.10)$ \\
\hline Green & $0.10(0.10)$ & $0.11(0.05)$ & $0.13(0.03)$ & $0.05(0.40)$ \\
\hline Brown & $0.01(0.90)$ & $-0.06(0.30)$ & $-0.05(0.40)$ & $-0.09(0.10)$ \\
\hline Pink & $0.03(0.60)$ & $0.00(1.00)$ & $0.08(0.20)$ & $-0.12(0.04)$ \\
\hline Tan & $0.00(0.90)$ & $-0.07(0.30)$ & $-0.05(0.40)$ & $-0.01(0.90)$ \\
\hline Red & $-0.02(0.70)$ & $-0.12(0.03)$ & $-0.10(0.07)$ & $-0.11(0.06)$ \\
\hline Purple & $-0.11(0.07)$ & $0.01(0.90)$ & $0.09(0.10)$ & $-0.06(0.40)$ \\
\hline Black & $-0.06(0.30)$ & $0.06(0.30)$ & $0.05(0.40)$ & $0.10(0.10)$ \\
\hline Blue & $-0.14(0.02)$ & $-0.06(0.30)$ & $-0.12(0.04)$ & $0.07(0.30)$ \\
\hline \multirow{2}{*}{$\begin{array}{l}\text { Green- } \\
\text { yellow }\end{array}$} & $-0.16(0.01)$ & $-0.10(0.07)$ & $-0.16(0.01)$ & $-0.03(0.60)$ \\
\hline & $\mathrm{Ag}$ & age & Node & \\
\hline
\end{tabular}

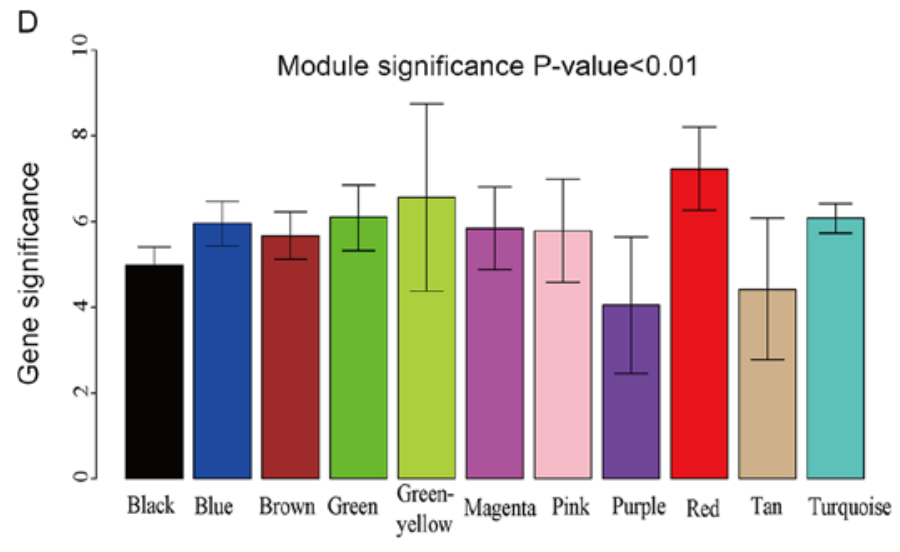

Figure 2. (A) Determination of soft-thresholding power in WGCNA. (B) Dendrogram of the genes modules based on a dissimilarity measure. (C) Heatmap of the correlation between module eigengenes and clinical characteristics of TNBC: Each row corresponds to a module eigengene and each column corresponds to a clinic characteristic. Each cell contains the corresponding correlation and P-value. The red key represents a positive correlation between modules and clinical variables, while the blue key represents opposite. (D) Distribution of mean gene significance and standard deviation in the modules associated with pathological stage of TNBC. TNBC, triple-negative breast cancer; WGCNA, weighted correlation network analysis.

clustering dendrograms that determined the generation of the co-expression modules. Then, the MEs were calculated by the moduleEigengenes function to quantify the co-expression similarity of the modules and the clustered modules were merged based on the similarity. A total of 11 distinct co-expression modules (ranging in size from 38-2,251 genes), were identified. These co-expression modules are represented by different colors in Fig. 2B. 
Table III. Comparison between non-relapse and relapse occurrence in patients with TNBC by Mann-Whitney U test.

\begin{tabular}{lccccc}
\hline Module & Ngene & Non-relapse & Relapse & Z score & P-value \\
\hline ME black & 848 & $-0.008(-0.03,0.02)$ & $-0.003(-0.03,0.04)$ & -1.579 & 0.114 \\
ME blue & 869 & $0.006(-0.03,0.03)$ & $0.001(-0.03,0.03)$ & -0.241 & 0.810 \\
ME brown & 654 & $-0.003(-0.04,0.03)$ & $-0.001(-0.03,0.03)$ & -0.297 & 0.766 \\
ME green & 338 & $-0.018(-0.03,0.01)$ & $-0.018(-0.03,0.03)$ & -0.434 & 0.665 \\
ME green yellow & 56 & $-0.008(-0.03,0.03)$ & $-0.011(-0.03,0.02)$ & -0.800 & 0.424 \\
ME magenta & 173 & $0.002(-0.03,0.04)$ & $-0.001(-0.04,0.02)$ & -1.099 & 0.272 \\
ME pink & 204 & $-0.009(-0.03,0.02)$ & $-0.007(-0.03,0.02)$ & -0.021 & 0.984 \\
ME purple & 75 & $-0.001(-0.03,0.03)$ & $-0.002(-0.03,0.02)$ & -0.565 & 0.572 \\
ME red & 276 & $0.007(-0.02,0.03)$ & $0.000(-0.03,0.02)$ & -2.393 & 0.017 \\
ME tan & 38 & $-0.005(-0.03,0.03)$ & $-0.002(-0.03,0.03)$ & -1.049 & 0.294 \\
ME turquoise & 2251 & $-0.008(-0.04,0.03)$ & $-0.012(-0.04,0.03)$ & -0.450 & 0.653 \\
\hline
\end{tabular}

Non-relapse and relapse describe the $\mathrm{P}_{50}\left(\mathrm{P}_{25}, \mathrm{P}_{75}\right)$ of MEs in each module. ME, module eigengenes; TNBC, triple-negative breast cancer; Ngene, number of genes identified in each module by WGCNA.
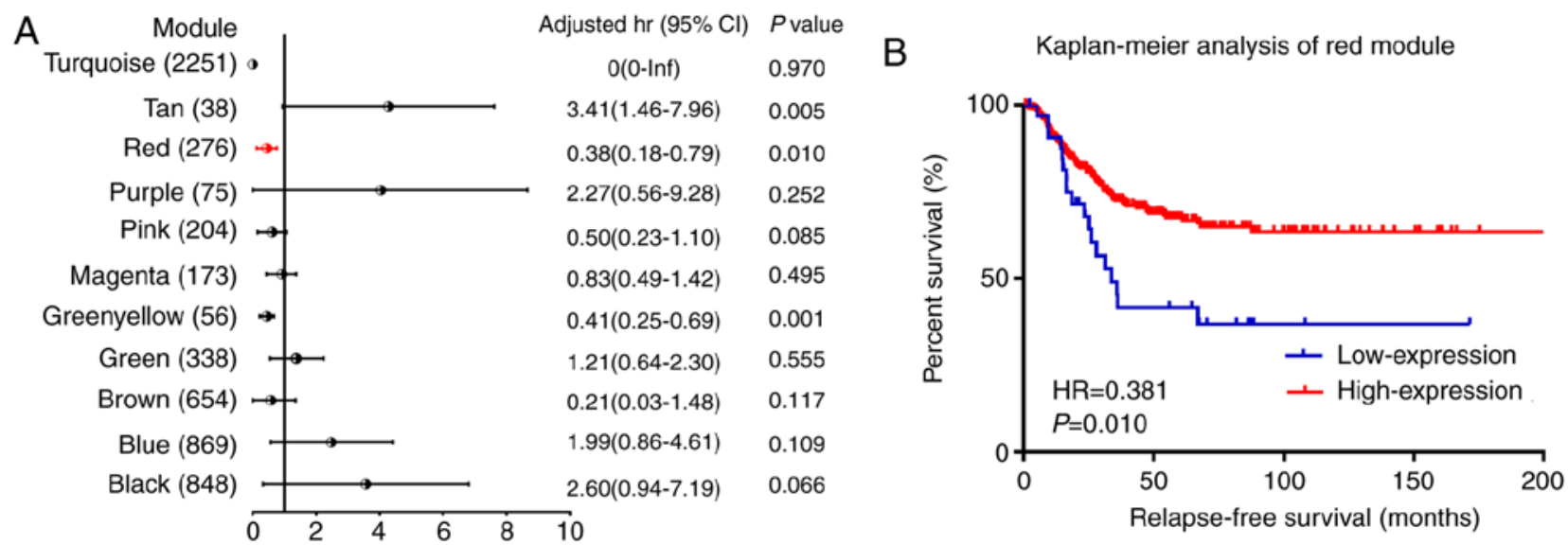

Figure 3. Survival analysis between module eigengenes and RFS in patients with TNBC. (A) Forest plot of hazard ratios in each module for RFS (B) Kaplan-Meier analysis of RFS for the red module. CI, confidence interval; HR, hazard ratio; RFS, relapse-free survival.

Identification of module associations with clinical characteristics of patients with TNBC. To determine the modules that were significantly associated with clinical characteristics, 294 TNBC samples with clinical variables were included to calculate the module-trait association (Fig. 2C). GS were also determined to evaluate the correlation between gene expression and pathological stage (Fig. 2D). Results of the module-trait association revealed a weak correlation between the red module and the pathological stage $(\mathrm{r}=-0.12$, $\mathrm{P}=0.03$ ) in which GS was the most significant. In addition, to identify associations between the co-expression modules and RFS endpoints, Cox regression was used to calculate the HRs and P-values for each ME. Following the survival analysis, 3 co-expression modules (green-yellow, red and tan colors) were significantly associated with the RFS in multivariate analysis (Fig. 3A). As presented in Fig. 3B, the increased mRNA expression of the red module was associated with good outcome for RFS, which was consistent with the correlation observed between pathological stage and ME. Furthermore, based on patients' status of relapse and non-relapse, each module was divided into two groups, and MEs were compared within these two groups with the Mann-Whitney U test. The results in Table III demonstrated that MEs in the red module were significantly different between the two groups of relapse and non-relapse patients with TNBC. These results allowed the selection of the red module as the module of interest, which was further studied in subsequent analyses.

Enrichment analysis of the key modules. GO and KEGG enrichment were performed in the red module using ClueGO. Following GO analysis of the red module, 40 GO terms were significantly enriched and were divided into 16 groups (Table IV), which reflected the biological processes. The top 10 enriched GO terms were defined as follows: 'mRNA processing', 'regulation of mitotic nuclear division', 'cellular response to topologically incorrect protein', 'interaction with symbiont', 'golgi vesicle transport', 'mitotic cytokinesis', 'regulation of target of rapamycin (TOR) signaling', 'transcription elongation from RNA polymerase II promoter', 'organelle 


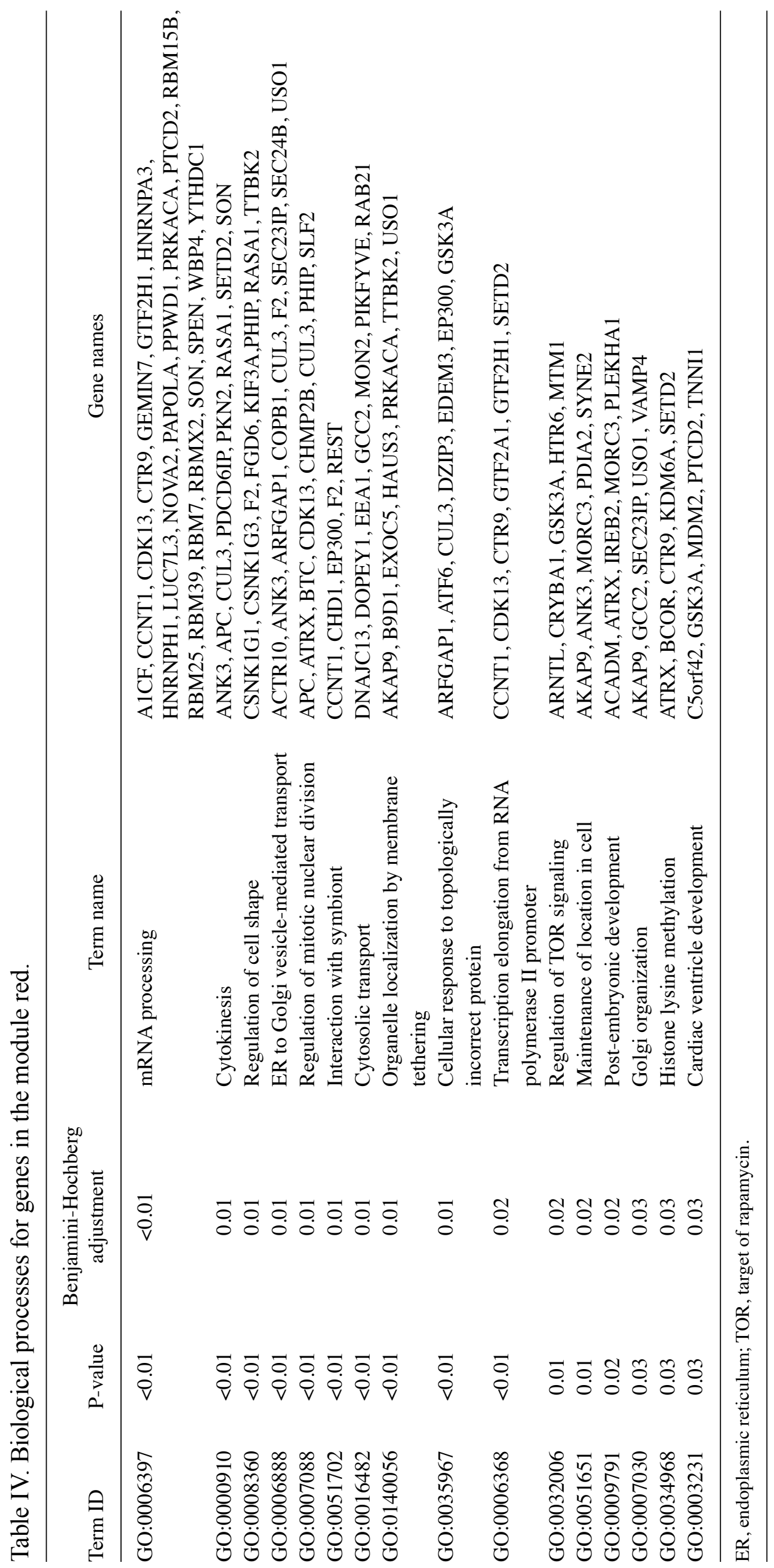


Table V. Survival analysis of hub genes for RFS in patients with TNBC.

\begin{tabular}{|c|c|c|c|c|c|c|c|}
\hline \multirow[b]{2}{*}{ Gene } & \multicolumn{3}{|c|}{ Univariate analysis $\left(413^{\mathrm{a}}\right)$} & \multicolumn{3}{|c|}{ Multivariate analysis $\left(294^{\mathrm{b}}\right)$} & \multirow[b]{2}{*}{ Cutoff value } \\
\hline & HR & P-value & $95 \% \mathrm{CI}$ & $\mathrm{HR}$ & P-value & $95 \% \mathrm{CI}$ & \\
\hline $\mathrm{APC}$ & 1.236 & 0.266 & $0.851-1.797$ & 1.064 & 0.800 & $0.658-1.721$ & -0.289 \\
\hline ATRX & 0.603 & 0.007 & $0.417-0.870$ & 0.601 & 0.033 & $0.376-0.960$ & 0.180 \\
\hline CHD1 & 0.643 & 0.120 & $0.368-1.122$ & 0.727 & 0.394 & $0.349-1.514$ & 0.949 \\
\hline CHD9 & 0.547 & 0.057 & $0.294-1.017$ & 0.373 & 0.033 & $0.151-0.925$ & 1.077 \\
\hline COL4A3BP & 0.609 & 0.015 & $0.406-0.911$ & 0.803 & 0.432 & $0.464-1.389$ & -0.725 \\
\hline $\mathrm{DCP} 2$ & 0.451 & 0.030 & $0.220-0.924$ & 0.471 & 0.060 & $0.215-1.032$ & 1.209 \\
\hline DMXL1 & 0.570 & 0.019 & $0.356-0.912$ & 0.608 & 0.094 & $0.339-1.089$ & 0.728 \\
\hline KIAA1033 & 0.793 & 0.203 & $0.554-1.134$ & 0.900 & 0.650 & $0.572-1.417$ & -0.930 \\
\hline RAPGEF6 & 0.687 & 0.063 & $0.462-1.021$ & 0.653 & 0.093 & $0.397-1.073$ & 0.328 \\
\hline TRIM23 & 0.361 & 0.015 & $0.159-0.821$ & 0.294 & 0.038 & $0.092-0.934$ & 1.187 \\
\hline TTC37 & 0.725 & 0.100 & $0.494-1.064$ & 0.654 & 0.100 & $0.395-1.085$ & 0.418 \\
\hline ZFYVE16 & 1.562 & 0.027 & $1.051-2.322$ & 1.309 & 0.304 & $0.783-2.188$ & -0.290 \\
\hline
\end{tabular}

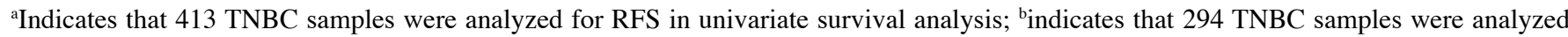
for RFS in multivariate analysis with adjustment of pathological stage. CI, confidence interval; HR, hazard ratio, TNBC, triple-negative breast cancer; RFS, relapse-free survival.

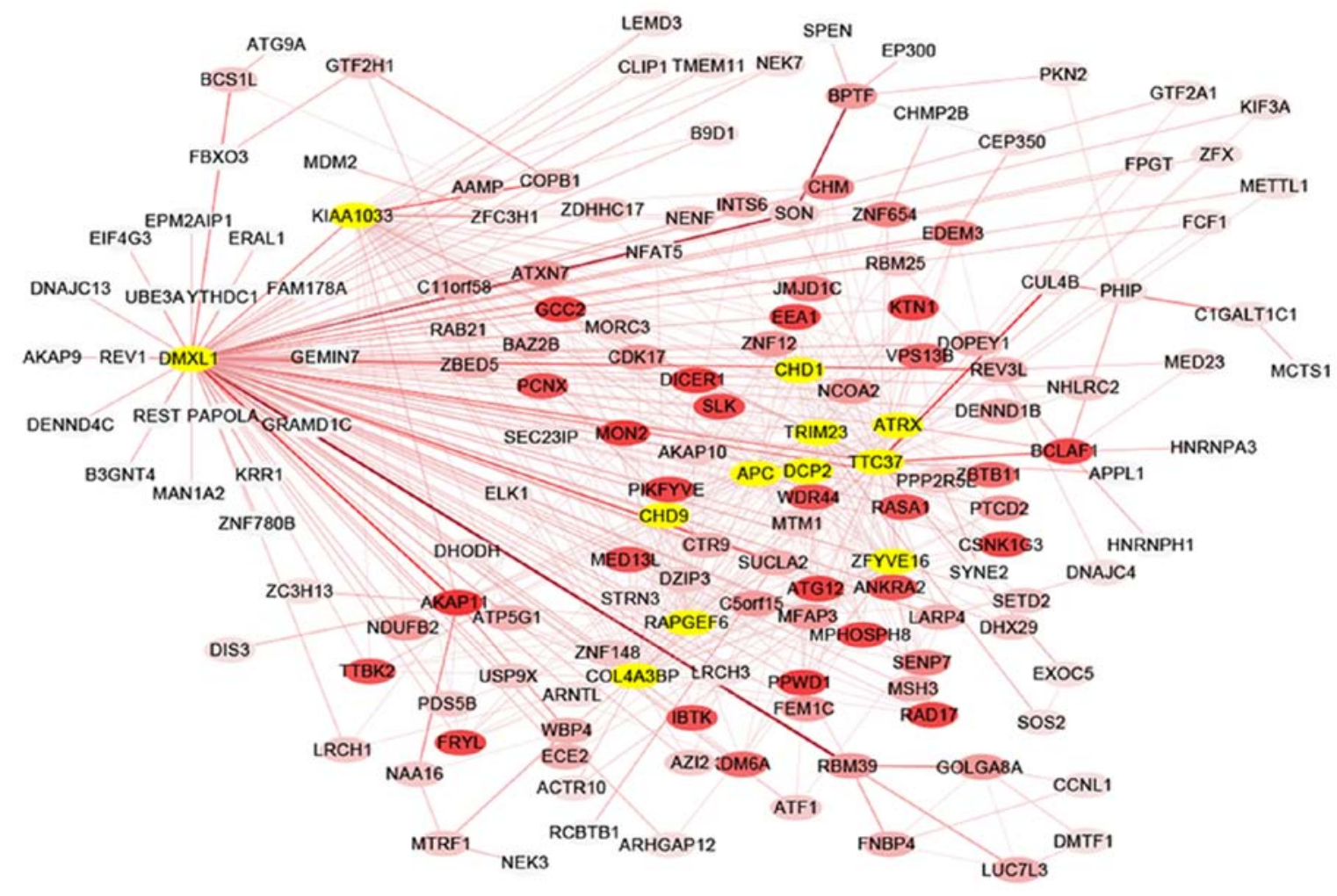

Figure 4. Visualization of the weighted gene correlation network in the red module: Cytoscape analysis identified 12 hub genes that are highlighted in bold and yellow.

localization by membrane tethering' and 'histone lysine methylation' Following KEGG analysis, 3 KEGG pathways were significantly identified, including the Hedgehog (Hh) signaling pathway (KEGG: 04340), the gonadotropin-releasing hormone (GnRH) signaling pathway (KEGG: 04912) and the thyroid hormone signaling pathway (KEGG: 04919).
Hub gene identification in the interested module. The co-expression network of selected hub genes from all genes in the red module were constructed with Cytoscape (Fig. 4). Following the measure of the absolute value of Pearson's correlation $(\mathrm{MM}>0.55), 26$ genes with higher connectivity were identified. Amongst these genes, those that were connected 


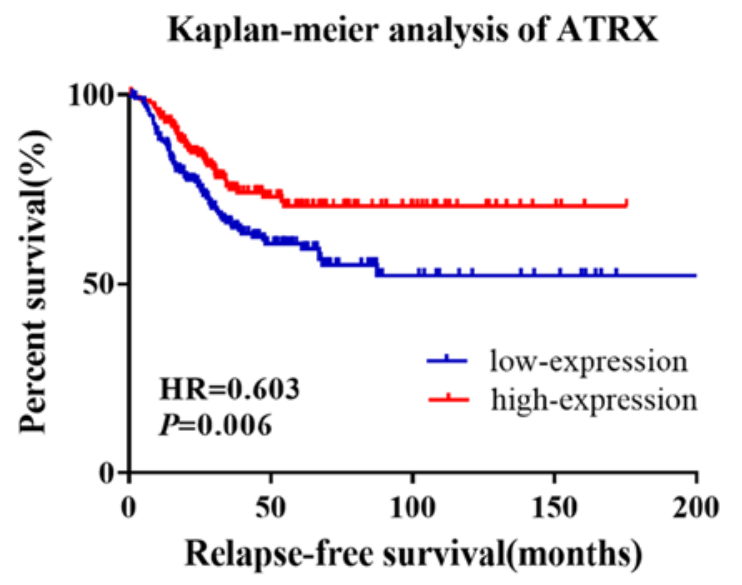

Kaplan-meier analysis of DCP2

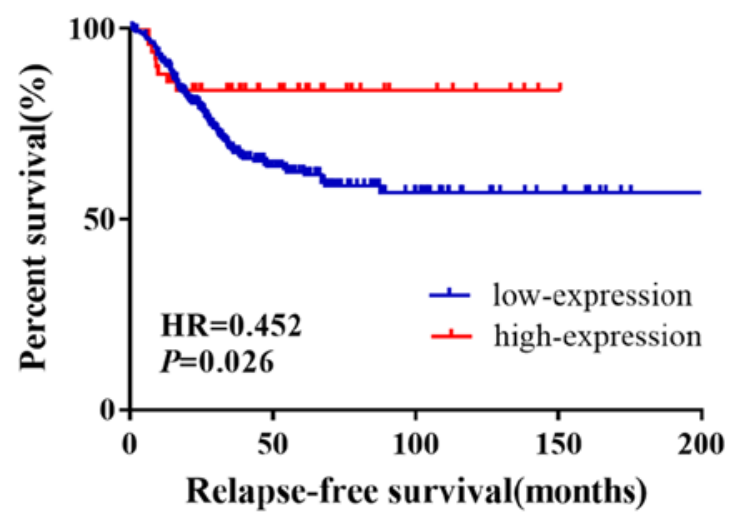

Kaplan-meier analysis of TRIM23

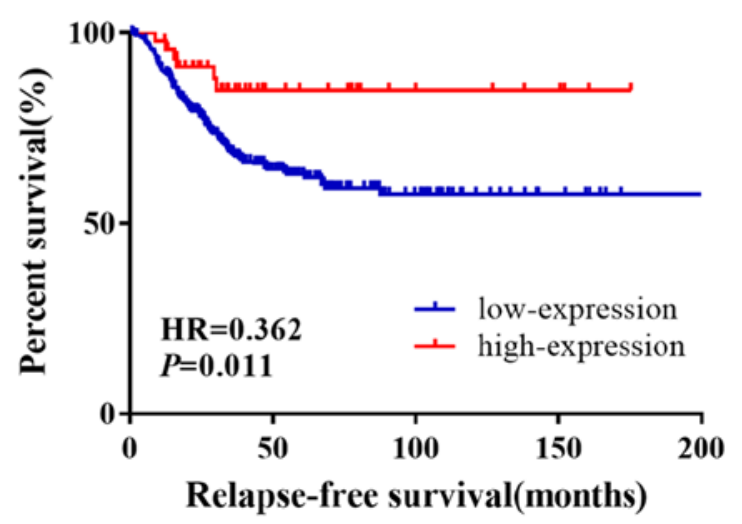

Kaplan-meier analysis of COL4A3BP

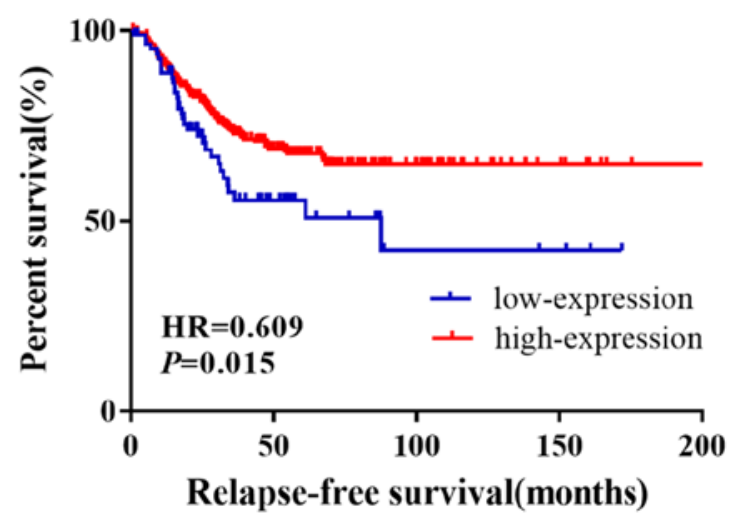

Kaplan-meier analysis of DMXL1
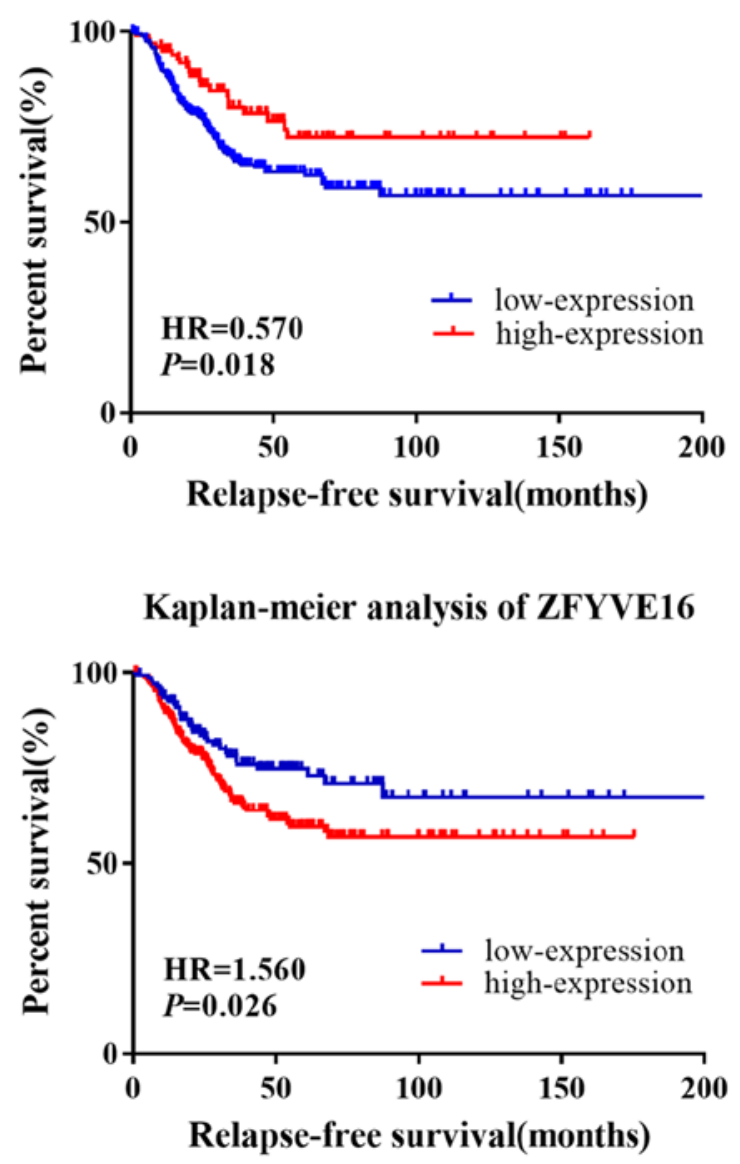

Figure 5. Association between mRNA expression of hub genes and relapse-free survival in patients with TNBC. HR, hazard ratio; TNBC, triple-negative breast cancer.

with $>15$ nodes were selected in the red module. A total of 12 common genes were eventually defined as hub genes and comprised WNT signaling pathway regulator (APC), $\alpha$ thalassemia/mental retardation syndrome X-linked (ATRX), chromodomain helicase DNA binding protein 1 (CHD1) and 9 (CHD9), collagen type IV $\alpha 3$ binding protein (COL4A3BP), decapping mRNA 2 (DCP2), Dmx like 1 (DMXL1), WASH complex subunit 4 (KIAA1033), Rap guanine nucleotide exchange factor 6 (RAPGEF6), tripartite motif containing 23 (TRIM23), tetratricopeptide repeat domain 37 (TTC37) and zinc finger FYVE-type containing 16 (ZFYVE16).
The HRs and P-values were calculated for the 12 genes in the survival analysis (Table V). Following the univariate survival analysis, ATRX, COL4A3BP, DCP2, DMXL1, TRIM23 and ZFYVE16 were found to be significantly associated with RFS in patients with TNBC (Fig. 5). The pathological stage variable in the multivariate survival analysis was adjusted and the result showed that ATRX, CHD9 and TRIM23 were significantly associated with RFS in patients with TNBC. To validate these three genes, 413 TNBC samples were divided into two groups based on the occurrence history of tumor relapse in patients with TNBC. 

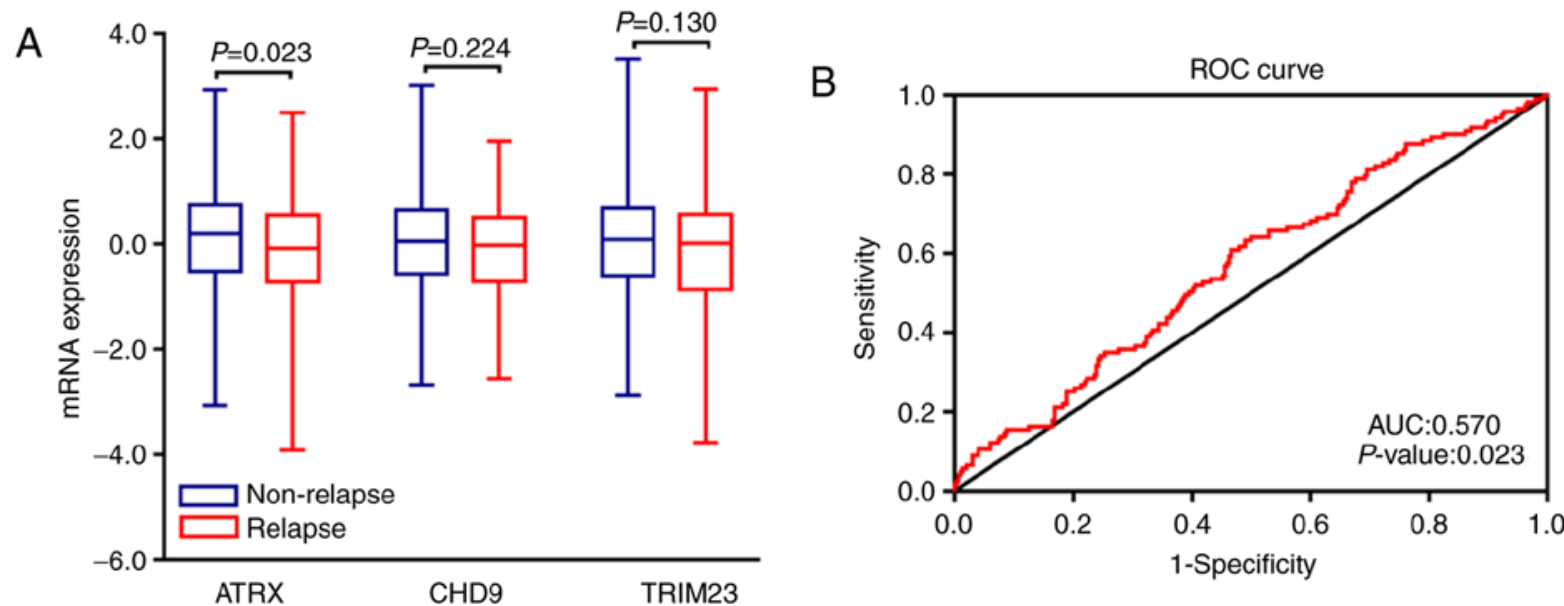

Figure 6. Comparison between non-relapse and relapse of hub genes in patients with TNBC. (A) Boxplots of mRNA expression in ATRX, CHD9 and TRIM23 between non-relapse and relapse groups of patients with TNBC: 413 TNBC samples were divided into two groups according to tumor relapse occurrence. (B) ROC curves: AUC was calculated to evaluate the diagnostic efficiency of ATRX to distinguish between relapse and non-relapse in patients with TNBC. CHD9, chromodomain helicase DNA binding protein 9; ATRX, $\alpha$ thalassemia/mental retardation syndrome X-linked; AUC, area under the curve; ROC, receiver operating characteristic; TNBC, triple-negative breast cancer; TRIM23, tripartite motif containing 23.

The independent sample Mann-Whitney U test was performed between two groups. The results suggested that the mRNA expression of ATRX could be used to distinguish the relapse from non-relapse in patients with TNBC (Fig. 6A). In addition, the ROC curve analysis further validated that ATRX may distinguish relapse from non-relapse in patients with TNBC $(\mathrm{P}=0.023$; AUC $=0.570$; Fig. 6B). Patients with lower mRNA expression of ATRX presented significantly shorter overall survival time, suggesting that ATRX may be considered as a prognosis biomarker of TNBC.

\section{Discussion}

In the present study, 11 co-expression modules were constructed based on the expression levels of 5,782 genes obtained from 456 patients with TNBC using the WGCNA method. WGCNA is a powerful method used to investigate biological mechanisms and identify genes in large-scale cancer gene expression datasets. The WGCNA method uses a soft threshold to weight the correlation between genes to determine the degree of association between them, which makes the co-expression network more consistent with biological network characteristics, and it provides results with high reliability and biological significance $(22,23)$. To the best of our knowledge, the analysis of TNBC mRNA expression using WGCNA has not yet been investigated. Results of survival analysis and WGCNA from the present study may therefore be considered as relevant for prognosis in TNBC.

With regards to the GO enrichment analysis, results demonstrated that the red module was associated with the biological processes involved in the regulation of intracellular signal activities, including the 'regulation of TOR signaling' and 'histone lysine methylation'. It has been reported that the phosphoinositide 3-kinase (PI3K)/protein kinase $\mathrm{B} /$ mechanistic (m)TOR pathway is essential in cell proliferation, metabolism, proliferation, differentiation, survival and angiogenesis, and in TNBC (24-27). As a key downstream component of the PI3K pathway, mTOR is a crucial regulator of tumor formation and progression. Crown et al (28) reported that targeting mTOR pathway inhibits tumor growth. mTOR inhibitors are being evaluated in patients with TNBC in clinical characteristics. In addition, histone lysine methylation is associated with nucleosome remodeling and gene expression regulation and is therefore considered as the key epigenetic process (29-31). Increasing evidence suggests that aberrant regulation of gene expression via histone methylation has emerged as an important mechanism for cancer initiation and progression $(30,32,33)$.

With regards to the KEGG pathway analysis, results demonstrated that the Hh signaling was the most significantly identified pathway. The Hh pathway serves a key role in embryonic development and regulates stem cell renewal and tissue homeostasis (34). It has been reported that dysregulated $\mathrm{Hh}$ signaling leads to increased aggressiveness of TNBC tumors, and that activation of $\mathrm{Hh}$ pathway enhances proliferation, invasion and migration of TNBC cells (35-37). Furthermore, the present study demonstrated that $\mathrm{GnRH}$ signaling was significantly identified in the red module. Effective therapies for patients with hormone-receptor-positive or HER2-positive breast cancer are available; however, treatments for TNBC are lacking (38). The present study demonstrated that the $\mathrm{GnRH}$, also known as luteinizing hormone-releasing hormone (LHRH), and its receptor may be involved in the negative regulation of cell proliferation in malignant tumors. Previous studies have reported that LHRH receptors are expressed in a significant proportion of TNBC, and are successfully targeted by cytotoxic LHRH analogs in vivo (38-42). Subsequently, further clinical trials using LHRH agonists in patients with TNBC may be considered in the future.

Hub genes, defined as highly connected genes in co-expression modules (14) are considered to serve important roles in the underlying mechanisms of malignancy, for example, uveal melanoma, colon cancer and human osteosarcoma $(17,43)$. In the present study, 12 hub genes were identified in the red module, including APC, ATRX, CHD1, CHD9, COL4A3BP, DCP2, DMXL1, KIAA1033, RAPGEF6, TRIM23, TTC37 and ZFYVE16. Notably, ATRX was significantly associated with RFS in TNBC samples, and Mann-Whitney U test 
demonstrated that ATRX mRNA expression may distinguish relapse from non-relapse occurrence in patients with TNBC. It has been reported that ATRX can modulate numerous cellular processes including transcription, DNA repair and mitotic recombination (44). In addition, an alternative lengthening of telomeres (ALT), one role of which is to maintain telomere lengths, has been detected in breast cancer (45), which suggests that ATRX may be a suppressor of ALT $(46,47)$. Furthermore, the loss of ATRX expression is associated with a poor prognosis and rapid tumor progression in melanoma, leiomyosarcomas and pancreatic neuroendocrine tumors (48-50). This may be of interest in the development of ALT-specific targets for TNBC treatment.

In conclusion, the present study attempted to explore potential molecular mechanisms in TNBC by using bioinformatics analyses. The red module identified has been associated with prognosis in TNBC, and functional analyses demonstrated that regulation of TOR signaling, histone lysine methylation and Hh signaling pathway may facilitate relapse and metastasis in patients with TNBC. In addition, the hub genes that were identified, including ATRX, may be considered as potential targets in TNBC. However, as the present study is mainly based on the analysis of five publicly available datasets, further detailed experimental research is required to confirm the results.

\section{Acknowledgements}

Not applicable.

\section{Funding}

No funding was received.

\section{Availability of data and materials}

The datasets analyzed during the current study are available in The Cancer Genome Atlas (cancergenome.nih.gov/) and the Gene Expression Ominibus database (ncbi.nlm.nih.gov/gds/).

\section{Authors' contributions}

YS and JJ designed the study and presented the results for group discussions. QC and LL collected the public datasets and preprocessed the data. QC, BX and LL provided methods and finished the description of the results for the manuscript. BX and LL programmed $\mathrm{R}$ codes and organized the manuscript. YS and $\mathrm{JJ}$ were responsible for the supervision and direction of all of the work. All authors have read and approved the final manuscript.

\section{Ethics approval and consent to participate}

Not applicable.

\section{Patient consent for publication}

Not applicable.

\section{Competing interests}

The authors declare that they have no competing interests.

\section{References}

1. Siegel RL, Miller KD and Jemal A: Cancer statistics, 2018. CA Cancer J Clin 68: 7-30, 2018.

2. Kumar P and Aggarwal R: An overview of triple-negative breast cancer. Arch Gynecol Obstet 293: 247-269, 2016.

3. Foulkes WD, Smith IE and Reis-Filho JS: Triple-negative breast cancer. N Engl J Med 363: 1938-1948, 2010.

4. Aysola K, Desai A, Welch C, Xu J, Qin Y, Reddy V, Matthews R, Owens C, Okoli J, Beech DJ, et al: Triple negative breast cancer-an overview. Hereditary Genet 2013 (Suppl 2): 001, 2013.

5. Saha P and Nanda R: Concepts and targets in triple-negative breast cancer: Recent results and clinical implications. Ther Adv Med Oncol 8: 351-359, 2016.

6. Xu YL, Yao R, Li J, Zhou YD, Mao F, Pan B and Sun Q: FOXC1 overexpression is a marker of poor response to anthracycline-based adjuvant chemotherapy in sporadic triple-negative breast cancer. Cancer Chemother Pharmacol 79: 1205-1213, 2017.

7. Horvath S, Zhang B, Carlson M, Lu KV, Zhu S, Felciano RM, Laurance MF, Zhao W, Qi S, Chen Z, et al: Analysis of oncogenic signaling networks in glioblastoma identifies ASPM as a novel molecular target. Proc Natl Acad Sci USA 103: 17402-17407, 2006.

8. Wang L, Tang H, Thayanithy V, Subramanian S, Oberg AL, Cunningham JM, Cerhan JR, Steer CJ and Thibodeau SN: Gene networks and microRNAs implicated in aggressive prostate cancer. Cancer Res 69: 9490-9497, 2009.

9. Zhang B and Horvath S: A general framework for weighted gene co-expression network analysis. Stat Appl Genet Mol Biol 4: Article17, 2005.

10. Zhao W, Langfelder P, Fuller T, Dong J, Li A and Hovarth S: Weighted gene coexpression network analysis: State of the art. J Biopharm Stat 20: 281-300, 2010.

11. Chen L, Yuan L, Wang Y, Wang G, Zhu Y, Cao R, Qian G, Xie C, Liu X, Xiao Y and Wang X: Co-expression network analysis identified FCER1G in association with progression and prognosis in human clear cell renal cell carcinoma. Int J Biol Sci 13: 1361-1372, 2017.

12. Wan Q, Tang J, Han Y and Wang D: Co-expression modules construction by WGCNA and identify potential prognostic markers of uveal melanoma. Exp Eye Res 166: 13-20, 2018.

13. Clarke C, Madden SF, Doolan P, Aherne ST, Joyce H, O'Driscoll L, Gallagher WM, Hennessy BT, Moriarty M, Crown J, et al: Correlating transcriptional networks to breast cancer survival: A large-scale coexpression analysis. Carcinogenesis 34: 2300-2308, 2013.

14. Langfelder $P$ and Horvath S: WGCNA: An R package for weighted correlation network analysis. BMC Bioinformatics 9: 559, 2008.

15. Langfelder $P$ and Horvath S: WGNCA: an R package for weighted correlation network analysis. BMC Genetics 9: 559, 2008.

16. Chen F, Zhu HH, Zhou LF, Li J, Zhao LY, Wu SS, Wang J, Liu W and Chen Z: Genes related to the very early stage of ConA-induced fulminant hepatitis: A gene-chip-based study in a mouse model. BMC Genomics 11: 240, 2010.

17. Liu X, Hu AX, Zhao JL and Chen FL: Identification of key gene modules for in human osteosarcoma by co-expression analysis weighted gene co-expression network analysis (WGCNA). J Cell Biochem 118: 3953-3959, 2017.

18. Yuan L, Chen L, Qian K, Wang G, Lu M, Qian G, Cao X, Jiang W, Xiao Y and Wang X: A novel correlation between ATP5A1 gene expression and progression of human clear cell renal cell carcinoma identified by co-expression analysis. Oncol Rep 39: 525-536, 2018.

19. Budczies J, Klauschen F, Sinn BV, Győrffy B, Schmitt WD, Darb-Esfahani S and Denkert C: Cutoff finder: A comprehensive and straightforward web application enabling rapid biomarker cutoff optimization. PLoS One 7: e51862, 2012.

20. Lotia S, Montojo J, Dong Y, Bader GD and Pico AR: Cytoscape app store. Bioinformatics 29: 1350-1351, 2013.

21. Bindea G, Mlecnik B, Hackl H, Charoentong P, Tosolini M, Kirilovsky A, Fridman WH, Pagès F, Trajanoski Z and Galon J: ClueGO: A Cytoscape plug-in to decipher functionally grouped gene ontology and pathway annotation networks. Bioinformatics 25: 1091-1093, 2009.

22. Lu X, Deng Y, Huang L, Feng B and Liao B: A co-expression modules based gene selection for cancer recognition. J Theor Biol 362: 75-82, 2014.

23. Deng SP, Zhu L and Huang DS: Mining the bladder cancer-associated genes by an integrated strategy for the construction and analysis of differential co-expression networks. BMC Genomics 16 (Suppl 3): S4, 2015. 
24. Montero JC, Esparís-Ogando A, Re-Louhau MF, Seoane S, Abad M, Calero R, Ocaña A and Pandiella A: Active kinase profiling, genetic and pharmacological data define mTOR as an important common target in triple-negative breast cancer. Oncogene 33: 148-156, 2014.

25. Pelicano H, Zhang W, Liu J, Hammoudi N, Dai J, Xu RH, Pusztai L and Huang P: Mitochondrial dysfunction in some triple-negative breast cancer cell lines: Role of mTOR pathway and therapeutic potential. Breast Cancer Res 16: 434, 2014.

26. Hatem R, El Botty R, Chateau-Joubert S, Servely JL, Labiod D, de Plater L, Assayag F, Coussy F, Callens C, Vacher S, et al: Targeting mTOR pathway inhibits tumor growth in different molecular subtypes of triple-negative breast cancers. Oncotarget 7: 48206-48219, 2016.

27. Massihnia D, Galvano A, Fanale D, Perez A, Castiglia M Incorvaia L, Listì A, Rizzo S, Cicero G, Bazan V, et al: Triple negative breast cancer: Shedding light onto the role of pi3k/akt/mtor pathway. Oncotarget 7: 60712-60722, 2016.

28. Crown J, O'Shaughnessy J and Gullo G: Emerging targeted therapies in triple-negative breast cancer. Ann Oncol 23 (Suppl 6): vi56-65, 2012

29. Paolicchi E, Crea F, Farrar WL, Green JE and Danesi R: Histone lysine demethylases in breast cancer. Crit Rev Oncol Hematol 86 97-103, 2013.

30. McGrath $\mathbf{J}$ and Trojer P: Targeting histone lysine methylation in cancer. Pharmacol Ther 150: 1-22, 2015.

31. Li LX, Zhou JX, Calvet JP, Godwin AK, Jensen RA and Li X: Lysine methyltransferase SMYD2 promotes triple negative breast cancer progression. Cell Death Dis 9: 326, 2018.

32. Copeland RA: Molecular pathways: Protein methyltransferases in cancer. Clin Cancer Res 19: 6344-6350, 2013.

33. McCabe MT, Mohammad HP, Barbash O and Kruger RG: Targeting histone methylation in cancer. Cancer J 23: 292-301, 2017.

34. Habib JG and O'Shaughnessy JA: The hedgehog pathway in triple-negative breast cancer. Cancer Med 5: 2989-3006, 2016.

35. O'Toole SA, Beith JM, Millar EK, West R, McLean A, Cazet A, Swarbrick A and Oakes SR: Therapeutic targets in triple negative breast cancer. J Clin Pathol 66: 530-542, 2013

36. Jamdade VS, Sethi N, Mundhe NA, Kumar P, Lahkar M and Sinha N: Therapeutic targets of triple negative breast cancer: A review. Br J Pharmacol 172: 4228-4237, 2015.

37. Di Mauro C, Rosa R, D'Amato V,Ciciola P, Servetto A,Marciano R, Orsini RC, Formisano L, De Falco S, Cicatiello V, et al: Hedgehog signalling pathway orchestrates angiogenesis in triple-negative breast cancers. Br J Cancer 116: 1425-1435, 2017.

38. SeitzS,BuchholzS,SchallyAV,WeberF,Klinkhammer-SchalkeM, Inwald EC, Perez R, Rick FG, Szalontay L, Hohla F, et al: Triple negative breast cancers express receptors for LHRH and are potential therapeutic targets for cytotoxic LHRH-analogs, AEZS 108 and AEZS 125. BMC Cancer 14: 847, 2014.

39. Buchholz S, Seitz S, Schally AV, Engel JB, Rick FG, Szalontay L, Hohla F, Krishan A, Papadia A, Gaiser T, et al: Triple-negative breast cancers express receptors for luteinizing hormone-releasing hormone (LHRH) and respond to LHRH antagonist cetrorelix with growth inhibition. Int J Oncol 35: 789-796, 2009.
40. Föst C, Duwe F, Hellriegel M, Schweyer S, Emons G and Gründker C: Targeted chemotherapy for triple-negative breast cancers via LHRH receptor. Oncol Rep 25: 1481-1487, 2011.

41. Buchholz S, Seitz S, Engel JB, Montero A, Ortmann O, Perez R, Block NL and Schally AV: Search for novel therapies for triple negative breast cancers (TNBC): Analogs of luteinizing hormone-releasing hormone (LHRH) and growth hormone-releasing hormone (GHRH). Horm Mol Biol Clin Investig 9: 87-94, 2012.

42. Kwok CW, Treeck O, Buchholz S, Seitz S, Ortmann O and Engel JB: Receptors for luteinizing hormone-releasing hormone $(\mathrm{GnRH})$ as therapeutic targets in triple negative breast cancers (TNBC). Target Oncol 10: 365-373, 2015.

43. Zhai X, Xue Q, Liu Q, Guo Y and Chen Z: Colon cancer recurrenceassociated genes revealed by WGCNA coexpression network analysis. Mol Med Rep 16: 6499-6505, 2017.

44. Shay JW, Reddel RR and Wright WE: Cancer. Cancer and telomeres-an ALTernative to telomerase. Science 336: 1388-1390, 2012.

45. Subhawong AP, Heaphy CM, Argani P, Konishi Y, Kouprina N, Nassar H, Vang R and Meeker AK: The alternative lengthening of telomeres phenotype in breast carcinoma is associated with HER-2 overexpression. Mod Pathol 22: 1423-1431, 2009.

46. Napier CE, Huschtscha LI, Harvey A, Bower K, Noble JR, Hendrickson EA and Reddel RR: ATRX represses alternative lengthening of telomeres. Oncotarget 6: 16543-16558, 2015.

47. Watson LA, Goldberg $\mathrm{H}$ and Bérubé NG: Emerging roles of ATRX in cancer. Epigenomics 7: 1365-1378, 2015.

48. Marinoni I, Kurrer AS, Vassella E, Dettmer M, Rudolph T, Banz V, Hunger F, Pasquinelli S, Speel EJ and Perren A: Loss of DAXX and ATRX are associated with chromosome instability and reduced survival of patients with pancreatic neuroendocrine tumors. Gastroenterology 146: 453-460.e5, 2014.

49. Qadeer ZA, Harcharik S, Valle-Garcia D, Chen C, Birge MB, Vardabasso C, Duarte LF and Bernstein E: Decreased expression of the chromatin remodeler ATRX associates with melanoma progression. J Invest Dermatol 134: 1768-1772, 2014.

50. Yang CY, Liau JY, Huang WJ, Chang YT, Chang MC, Lee JC, Tsai JH, Su YN, Hung CC and Jeng YM: Targeted next-generation sequencing of cancer genes identified frequent TP53 and ATRX mutations in leiomyosarcoma. Am J Transl Res 7: 2072-2081, 2015.

This work is licensed under a Creative Commons Attribution-NonCommercial-NoDerivatives 4.0 International (CC BY-NC-ND 4.0) License. 